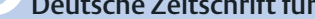 Osteopathie}

Offizielles Organ folgender Verbände

Verband der Osteopathen Deutschland e.V. (VOD)

Deutsche Akademie für Osteopathische Medizin e.V. (DAOM)

Dssociation Luxembourgeoise des Ostéopathes (A.L.D.O.)

ISSN 1610-5044

$2003 ; 1(2)$

Herausgeber :

Prof. Dr. rer. nat. med. habil. Rainer Breul D.O.h.c.

Marina Fuhrmann, D.O. M.R.O.

Prof. Dr. med. habil. Karl-Ludwig Resch

Dr. med. Roger Seider, D.O.

Verlag

Hippokrates Verlag in

MVS Medizinverlage Stuttgart GmbH \& Co.KG

Oswald-Hesse-Str. 50, 70469 Stuttgart, Fax: (07 11) 8931-706 Geschäftsführung:

Dr. med. Thomas Scherb, Dipl. Kaufm. Albrecht Hauff

Programmplanung:

Marina Horbatsch

Fon/Fax: (07 11) 8931-715/-705

E-Mail: marina.horbatsch@medizinverlage.de und

redaktion.do@medizinverlage.de

Redaktion:

Christoph Newiger in team 4 U Medienbüro

Fon/Fax: (089) 209001-67/-68

E-Mail: redaktion.do@medizinverlage.de

Beratung:

Dorothee Seiz

in MVS Medizinverlage Stuttgart GmbH \& Co. KG

E-Mail: redaktion.do@medizinverlage.de

Marketing:

Marion Krubasik

Fon/Fax: (07 11) 89 31-735/-706

E-Mail: marion.krubasik@medizinverlage.de

Anzeigen:

Günter Fecke, MVS Media-Service

Fon/Fax: (07 11) 8931-714/-706

E Mail: guenter.fecke@medizinverlage.de

Abonnenten-Service

Fon/Fax: (07 11) 8931-321/-422

E Mail: kundenservice@thieme.de

Produktion

Satz und Gestaltung: Fotosatz Sauter, Donzdorf

Druck: Rondo Druck, Ebersbach

Autorenhinweise

Auf Anfrage bei der Redaktion.

Urheberrecht:

Mit der Annahme eines Manuskriptes erwirbt der Verlag für die Dauer der gesetzlichen Schutzfrist ( $\$ 64$ UrhRG) die ausschließliche Befugnis zur Wahrnehmung der Verwertungsrechte im Sinne der $\S \S 15 \mathrm{ff}$. des Urheberrechtsgesetzes. Die Zeitschrift und alle in ihr enthaltenen einzelnen Beiträge und Abbildungen sind für die Dauer des Urheberrechts geschützt. Jede Verwertung ist ohne Zustimmung des Verlages außerhalb der engen Grenzen des UrhRG unzulässig und strafbar. Dies gilt insbesondere für Vervielfältigungen, Übersetzungen, Microverfilmungen und die Einspeicherung und Verarbeitung in elektronischen Systemen. Für den persönlichen Gebrauch dürfen von Beiträgen oder Teilen von diesen einzelne Kopien hergestellt werden. Die Rechte an den Abbildungen liegen

- wenn nicht anders gekennzeichnet - beim Verlag.

Erscheinungsweise:

Vierteljährlich

Bezugspreise

preis

Inland $€ 64,9$

Europa $€ 64,90$

$€ 64,90$

Versand-

kosten

Gesamt

$€ 5,90$

$€ 70,80$

Restl. Welt

$€ 10,40$

$€ 75,30$

Aus-, Fort- und Weiterbildungspreis/Vorzugspreis für Studenten und AIP:

$\begin{array}{llll}\text { Inland } & € 44,90 & € 5,90 & € 50,80 \\ \text { Europa } & € 44,90 & € 10,40 & € 55,30 \\ \text { Restl. Welt } & € 44,90 & € 18,90 & € 63,80\end{array}$

Einzelheft $€ 18,00$ zzgl. Versandkosten ab Verlagsort. Alle

Preise sind unverbindlich empfohlene Preise. Alle Preise und Versandspesen enthalten 7\% MwSt.

\section{Was denken sich eigentlich diese Herausgeber?}

$\mathrm{D}$ a haben wir mächtig Staub aufgewirbelt mit unserem Editorial aus der Kongressnummer zum Thema „Philosophie“. Bewusste Provokation unserer neuen Leserschaft? Keineswegs, sondern vielmehr Denkanstoss und Einladung, Position zu beziehen, die eigene Meinung kund zu tun und zwischen den Zeilen zu lesen. Ist es in der Osteopathie nicht auch so? „Lesen“ wir nur das, was uns der Organismus des Patienten vordergründig mit seinen Beschwerden zeigt? Oder sind wir nicht vielmehr gezwungen, wenn wir osteopathisch erfolgreich arbeiten wollen, „zwischen den Zeilen zu lesen“, um Zusammenhänge aufzudecken und zu den Ursachen von Beschwerden zu gelangen?

Über die Osteopathie lässt sich viel philosophieren, aber eben nicht im Sinne einer Geisteswissenschaft, sondern in Hinblick auf jene Philosophie, die den konzeptionellen Ansatz der Osteopathie ausmacht. Allzu leicht lässt sich das englische Wort „Philosophy“ im Deutschen mit „Philosophie“ übersetzen. Die Begriffe sind jedoch nicht deckungsgleich. Der englische Ausdruck lässt sich auch mit „praktische Lebensweisheit“, „Welt- oder Lebensanschauung“ übersetzen. In diesem Sinne hat wohl auch A.T. Still von „Philosophy“ gesprochen.

Das Bewusstsein um diese Weltanschauung bestimmt den Unterschied zu einer vermeintlich osteopathischen Behandlung, die nach Rezepten arbeitet und nur Techniken aneinander reiht. Wir stehen nicht für eine solche Form der Osteopathie. Das ist keine Osteopathie im Sinne Stills.

Gern blicken wir über den großen Teich, dabei lässt sich die Situation der Osteopathie in den USA mit der unseren nur sehr schwer vergleichen. $\mathrm{Zu}$ verschieden sind die Arbeitsbedingungen, die rechtliche Lage und die Entwicklungsgeschichte. Unsere Wurzeln liegen hier. Die Geschichte der Osteopathie in Deutschland und „good old Europe" ist überwiegend eine nichtärztliche. Genau das ist unser großer Vorteil, denn wir können nicht verführt werden durch eine Medizin, die vorwiegend Medikamente oder Apparate nutzt. Seien wir uns dessen bewusst - auch und gerade wenn es um die Etablierung des Osteopathen als eigenständigen Beruf geht.

Dennoch wird es in Deutschland auch weiterhin osteopathisch tätige Ärzte geben. Keine der Entwicklungen ist mehr rückgängig zu machen.

Wo stehen wir also? Wo steht jeder einzelne unserer Leser? Unsere Zeitschrift und das Editorial im Besonderen will die Grundlagen dazu liefern, über genau diese Themen nachzudenken. Ohne Polemik aber pointiert, nicht um negative Ideen zu etablieren, sondern um Konstruktives zu schaffen.

Sie als unsere kritischen Leser wollen wir dazu einladen, sich an diesem Diskurs zu beteiligen.

Dabei halten wir es mit Gotthold Ephraim Lessing: „Nicht die Wahrheit, in deren Besitz irgendein Mensch ist oder zu sein vermeint, sondern die aufrichtige Mühe, die er angewandt hat, hinter die Wahrheit zu kommen, macht den Wert des Menschen.“

\section{Die Herausgeber}

Titelbild:

E. Blechschmidt, Die pränatalen Organsysteme des Menschen.

Hippokrates 1973; Bearbeitung: Pdesign A. Page, Stuttgart 2002 